\title{
Structural features underlying T-cell receptor sensitivity to concealed MHC class I micropolymorphisms
}

\author{
Guillaume B. Stewart-Jones ${ }^{a}$, Peter Simpson ${ }^{a}$, P. Anton van der Merwe ${ }^{b}$, Philippa Easterbrook, ${ }^{c, d}$ \\ Andrew J. McMichael ${ }^{\mathrm{a}}$, Sarah L. Rowland-Jones ${ }^{\mathrm{a}}$, E. Yvonne Jones ${ }^{\mathrm{e}}$, and Geraldine M. Gillespie ${ }^{\mathrm{a}, 1}$
}

${ }^{a}$ Medical Research Council Human Immunology Unit, Weatherall Institute of Molecular Medicine, John Radcliffe Hospital, University of Oxford, Oxford OX3 9DS, United Kingdom; 'bir William Dunn School of Pathology, University of Oxford, Oxford OX1 3RE, United Kingdom; 'Western Education Centre, Kings College London School of Medicine and Dentistry, London SE5 9RJ, United Kingdom; 'Infectious Disease Institute, Makerere University,

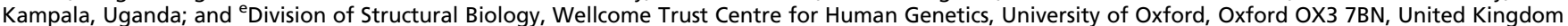

Edited by Philippa Marrack, Howard Hughes Medical Institute, National Jewish Health, Denver, CO, and approved October 9 , 2012 (received for review June 15, 2012)

\begin{abstract}
Polymorphic differences distinguishing MHC class I subtypes often permit the presentation of shared epitopes in conformationally identical formats but can affect T-cell repertoire selection, differentially impacting autoimmune susceptibilities and viral clearance in vivo. The molecular mechanisms underlying this effect are not well understood. We performed structural, thermodynamic, and functional analyses of a conserved T-cell receptor (TCR) which is frequently expanded in response to a HIV-1 epitope when presented by HLA-B 5701 but is not selected by HLA-B* 5703 , which differs from HLA-B* 5701 by two concealed polymorphisms. Our findings illustrate that although both HLA-B 57 subtypes display the epitope in structurally conserved formats, the impact of their polymorphic differences occurs directly as a consequence of TCR ligation, primarily because of peptide adjustments required for TCR binding, which involves the interplay of polymorphic residues and water molecules. These minor differences culminate in subtype-specific differential TCR-binding kinetics and cellular function. Our data demonstrate a potential mechanism whereby the most subtle MHC class I micropolymorphisms can influence TCR use and highlight their implications for disease outcomes.
\end{abstract}

$T^{1}$ he MHC class I (MHCI) locus is described consistently as a major host factor influencing disease outcome in the setting of HIV-1 infection (1). As MHCI molecules select the repertoires of viral epitopes presented to $\mathrm{CD}^{+} \mathrm{T}$ cells, they shape the immune response against the virus. A broad variety of distinct $\mathrm{MHCI}$ allotypes, which can demonstrate up to $10 \%$ amino acid diversity, allows extensive sampling of epitope repertoires, and these differences also influence the efficacy of viral control, as illustrated by the strong association of individual MHCI types with prolonged AIDS-free survival $(1,2)$. Less understood is the role of minor polymorphisms (termed "micropolymorphisms") that distinguish closely related MHCI subtypes, often by as few as one amino acid change. Although such minimal changes frequently allow identical epitopes to be presented, they often influence the efficacy of viral clearance (3) and disease susceptibilities in vivo (4). These effects have important implications in the context of HIV-1 infection, where the delicate interplay between $\mathrm{CD}^{+} \mathrm{T}$-cell selection, viral evolution, and fitness cost is assumed to shape the clinical course of disease (5).

MHCI subtypes separated by micropolymorphisms frequently permit the presentation of shared epitopes, however, these differences can affect the conformation of peptides in their binding grooves (6-8) and/or the positioning of MHC $\alpha 1 / \alpha 2$ helices $(9,10)$, with implications for T-cell receptor (TCR) usage in vivo. The protective HLA-B*5703 and B*5702 subtypes, for example, are distinguished by a single amino acid substitution that does not notably alter their peptide-binding motifs. However, the positioning of this polymorphism within the TCR footprint is likely to affect the use of the TCR repertoire and could explain the greater association of HLA-B*5703 with lower viral set point and immune control (11). Yet for other MHCI subtype-peptide combinations, minor polymorphic differences result in minimal, if any, conformational disparities (4) and the molecular processes underlying differential T-cell selection are not well understood. This is especially true for longer epitopes, which comprise an important group of ligands $(12,13)$ for which the diversity of their responding TCR repertoires may be further limited by their atypical structural conformations when presented by MHCI. Notable examples are the HLA-B*57 subtypes HLA-B*5701 and B*5703, which consistently are associated with prolonged AIDS-free survival (1416). Despite two polymorphic amino acids distinguishing these subtypes at residues 114 (D-N) and 116 (S-Y), both bind an equivalent repertoire of peptides (17) and in HIV-1 infection share similar $\mathrm{CD}^{+} \mathrm{T}$-cell immunodominance hierarchies from acute infection through to chronic disease (18-22). The contribution of these HLA-B*57 subtypes to successful viral control is thought to relate to the epitopes selected, most notably to three p24-derived capsid epitopes targeted. Two of these, the ISPRTLNAW (IW9) and TSTLQEQIGW (TW10) epitopes, are targeted in early infection, presumably contributing to rapid viral control $(22,23)$. The nature of T-cell-driven escape mutations that accrue for these epitopes are conserved in the presence of both B*57 subtypes, presumably reflecting shared modes of peptide presentation and shared $\mathrm{T}$-cell recognition conformations in vivo. However, the subtype-specific differences appear to impact a third epitope, KAFSPEVIPMF (KF11), which dominates the B*57restricted immune response in chronic disease $(18,21)$. Targeting of this epitope is important in patients in whom circulating IW9 and TW10 mutations lead to immune escape $(20,24,25)$, and its recognition is associated with lower plasma viral load (26). In HLA-B $* 5703^{+}$patients, diverse KF11 variants circulate, which frequently associate with elevated viral loads $(20,27)$. However, viruses harboring these mutations are rare in carriers of HLA-

Author contributions: G.B.S.-J. and G.M.G. designed research; G.B.S.-J., P.S., P.A.v.d.M., E.Y.J., and G.M.G. performed research; P.E., A.J.M., S.L.R.-J., and E.Y.J. contributed new reagents/analytic tools; G.B.S.-J., P.A.v.d.M., and G.M.G. analyzed data; and G.B.S.-J. and G.M.G. wrote the paper.

The authors declare no conflict of interest.

This article is a PNAS Direct Submission.

Data deposition: Crystallography, atomic coordinates, and structure factors reported in this paper have been deposited in the Protein Data Bank (PDB), www.pdb.org (PDB ID code 2 YPK for the HLA-B*5701-KF11 structure and 2YPL for the HLA-B*5703-KF11-AGA1 TCR structure)

${ }^{1}$ To whom correspondence should be addressed. E-mail: geraldine.gillespie@imm.ox.ac. uk.

See Author Summary on page 20190 (volume 109, number 50).

This article contains supporting information online at www.pnas.org/lookup/suppl/doi:10. 1073/pnas.1207896109/-/DCSupplemental. 
B*5701 $(24,28)$, a finding not readily explained by factors specific to the infecting viral clades (27). We and others have analyzed the KF11-specific TCR repertoire in HLA-B $* 57^{+}$patients and have reported common use of a conserved and frequently immunodominant $\mathrm{V} \alpha 5 / \mathrm{V} \beta 19$ TCR pair sharing highly conserved CDR3 $\alpha$ and $-\beta$ motifs in unrelated HLA-B* $5701^{+}$individuals $(21,27,29)$. This "public" TCR displays cross-reactivity against broad KF11 variants (30). However, this receptor pair does not represent a KF11specific clonotype in carriers of HLA-B*5703 and its absence might contribute to the higher incidence of circulating KF11 variants in HLA-B $* 5703^{+}$individuals $(21,27,31)$.

Although the majority of MHCI restricted epitopes are 8-10 amino acids, peptides of noncanonical length up to 13 residues, and particularly viral peptides targeted in humans, represent an important category of epitopes, (32-34). Longer peptides bind MHCI molecules either by extending beyond the peptide-binding groove (PBG) (35) or, more frequently, by forming a central bulge that arches above the cleft while maintaining standard A, B, and F pocket binding $(13,33,34,36)$. Although many arched peptides remain mobile (33), others assume a rigid conformation due to stabilizing interactions that involve hydrophobic forces (36) and water-mediated and direct peptide-MHC hydrogen bonds (9). Having previously determined the structure of KF11 in complex with HLA-B*5703, we observed a central peptide bulge, with two $P$ residues forming the basis of the stable peptide arch (36). Here we present a structural, thermodynamic, and functional study describing the molecular features underlying TCR-mediated recognition of the atypical KF11 epitope and highlight the vital role played by germline-encoded TCR $\alpha$-chain residues. We also demonstrate how minimal differences between the HLA-B*57 subtypes involving subtle alterations in the interplay of polymorphic residues and specific networks of water molecules in the PBG are paramount in facilitating optimal TCR binding, influencing the kinetics of the TCR-pMHCI interactions and cellular function. Collectively, our findings illustrate how subtle subtype-specific polymorphic differences can have important implications for T-cell use, repertoire diversity, and presumably, disease outcomes.

\section{Results}

Crystal Structures of B*5701-KF11 and AGA1-B*5703-KF11. The V $\alpha 5 /$ V $\beta 19$ (AGA1) TCR encoding the conserved CDR3 $\alpha$ and $-\beta$ motifs (V $\alpha 5$-SAIYFCALSGGYQKVTFGTGTKL and V $\beta 19$ TAFYLCASTGSYGYTFGSGTR) and HLA-B*5703 were refolded separately, purified, and crystallized as a complex. Refolded and purified HLA-B*5701-KF11 also was crystallized separately. X-ray diffraction datasets were collected and phased by molecular replacement, and structures were refined for the AGA1-HLA-B*5703-KF11 complex [2.4 $\AA$, crystallographic R index $\left(\mathrm{R}_{\text {cryst }}\right)=22.5 \%$, free $\mathrm{R}$-factor $\left.\left(\mathrm{R}_{\text {free }}\right)=29.7 \%\right]$ and the HLA-B*5701-KF11 complex $\left(1.95 \AA, \mathrm{R}_{\text {cryst }}=19.0 \%, \mathrm{R}_{\text {free }}=\right.$ $25.0 \%$ ). The unliganded peptide-MHC (pMHC) and TCR-pMHC complex crystal structures had one pMHC or complex, respectively, per crystallographic asymmetric unit. Lattice contacts did not involve the peptide in the HLA-B*5701-KF11 structure, thus providing unbiased views of the epitope structure and the associated hydration shell (Table 1). We previously reported the crystal structure of B*5703-KF11 (36). Unfortunately, we were unable to produce a diffracting HLA-B*5701-KF11-TCR complex; therefore we compared the structures of the pre- and post-TCR-bound states of the HLA-B*5703-KF11 epitope as a model to characterize the key structural determinants of the TCR-pMHC interaction. Based on the findings of this analysis, we probed the possible molecular origins of the biased TCR selection by comparison with the unliganded structure of HLA-B*5701-KF11.

Overall, the AGA1 TCR docks to the HLA-B*5703-KF11 in a diagonal manner similar to that described for other class I TCRpMHC complex structures (37), with the $\mathrm{V} \alpha / \mathrm{V} \beta$ axis of the TCR orientated $37^{\circ}$ to the peptide axis (Fig. $1 A$ and $B$ ). The total buried
Table 1. Crystallographic statistics

\begin{tabular}{|c|c|c|}
\hline Data collection & $B * 5701-K F 11$ & AGA1-B*5703-KF11 \\
\hline Space group & $P 2_{1} 2_{1} 2_{1}$ & $P 2_{1} 2_{1} 2_{1}$ \\
\hline \multicolumn{3}{|l|}{ Unit cell } \\
\hline Dimensions $(\AA ̊)(a, b, c)$ & $50.8,82.1,110.5$ & $56.3,75.6,241.5$ \\
\hline Angles $\left(^{\circ}\right)(\alpha, \beta, \gamma)$ & $90,90,90$ & $90,90,90$ \\
\hline Source & ID14 eh2 & ID14 eh2 \\
\hline \multicolumn{3}{|l|}{ Resolution $(\AA)$} \\
\hline Highest-resolution shell & $30-1.95(2.02-1.95)^{*}$ & $30-2.4(2.51-2.4)^{*}$ \\
\hline Measured reflections & 102,868 & 538,769 \\
\hline Unique reflections & 34,422 & 41,023 \\
\hline Completeness (\%) & $99.8(99.6) *$ & $99.0(94.7)^{*}$ \\
\hline $\mathrm{I} / \sigma(\mathrm{I})$ & $19.4(3.4) *$ & $40.1(5.5)^{\star}$ \\
\hline$R_{\text {merge }}(\%)^{\dagger}$ & $5.4(31.1)^{*}$ & $5.4(36.2) *$ \\
\hline \multicolumn{3}{|l|}{ Refinement statistics } \\
\hline Resolution range $(\AA)$ & $30-1.95(2.02-1.95)^{*}$ & $30-2.4(2.51-2.4) *$ \\
\hline $\mathrm{R}_{\text {cryst }}{ }^{\ddagger}$ & 19.1 & 22.1 \\
\hline $\mathrm{R}_{\text {free }} \S$ & 25.2 & 27.7 \\
\hline Number of residues & 384 & 931 \\
\hline $\begin{array}{l}\text { Number of water } \\
\text { molecules }\end{array}$ & 494 & 198 \\
\hline \multicolumn{3}{|l|}{ Rmsd from ideal } \\
\hline Bond lengths $(\AA ̊)$ & 0.012 & 0.007 \\
\hline Bond angles $\left({ }^{\circ}\right)$ & 1.54 & 1.22 \\
\hline \multicolumn{3}{|l|}{ Ramachandran plot (\%) } \\
\hline Favored & 92.8 & 92.0 \\
\hline Allowed & 6.6 & 5.9 \\
\hline Generous & 0.6 & 1.8 \\
\hline Disallowed & 0.0 & 0.3 \\
\hline
\end{tabular}

*Numbers in parentheses correspond to the outermost shell of data.

${ }^{\dagger} R_{\text {merge }}=\sum_{\text {hkl }}|\mathrm{I}-<\mathrm{l}\rangle \mid / \sum_{\mathrm{hkl}} I$ where I is the intensity of unique reflection $\mathrm{hkl}$ and $\langle\mathrm{l}\rangle$ is the average over symmetry-related observations of unique reflection hkl.

${ }^{ \pm} R_{\text {cryst }}=\sum\left|F_{\text {obs }}-F_{\text {calc }}\right| / \sum F_{\text {obs }}$ where $F_{\text {obs }}$ and $F_{\text {calc }}$ are the observed and calculated structure factors, respectively.

${ }^{\S} R_{\text {free }}$ is calculated as for $R_{\text {cryst }}$ but using $5 \%$ of reflections sequestered before refinement.

surface area (BSA) on complex formation is $1,600 \AA^{2}$, which is relatively low compared with other TCR-pMHC complexes. The TCR $\alpha$-chain dominates the interaction with pMHC, contributing $71 \%$ of the BSA, the largest fraction observed for a TCR-pMHCI complex published to date. Dominant TCR $\alpha$-chain involvement has been described previously for the HLA-A*0201-Tax-specific A6 and B7 TCRs and for the B*3508-resticted SB27 TCR (9), with $65 \%, 69 \%$, and $58 \%$ of total BSA contributions, respectively, conferred by the $\alpha$-chain. In contrast, in the JM22-HLA-A*0201flu complex $67 \%$ of the BSA is contributed by the V $\beta 19$ chain, which interestingly is the same V $\beta$ chain used by the AGA1 TCR (38).

The bulged structure of KF11 is accommodated in a large cavity formed between the CDR $1 \alpha, \mathrm{CDR} 3 \alpha$, and CDR $3 \beta$ loops, whereas the MHC helices are contacted by the CDR $1 \alpha,-2 \alpha,-3 \alpha-$, and $-3 \beta$ loops. The V $\beta 19$ CDR1 and CDR2 framework regions are largely solvent exposed and make little contact with pMHC (Fig. $1 C$ and $D$ and Tables 2-5). The buried pMHC surface area in the HLAB*5703-KF11-AGA1 complex structure is apportioned $52 \%$ to the MHC and $48 \%$ to the peptide. This configuration resembles the HLA-B*3508-SB27 TCR cocomplex topology (9) and is consistent with the large surface area of KF11 contacted by the TCR but is in contrast with the majority of TCR-pMHC complexes, in which a much greater proportion of the total BSA is apportioned to the MHC.

The AGA1 TCR has comparatively short CDR3 loops but recognizes this bulged peptide by clamping the crest of the peptide, extending the CDR3 $\alpha$ Y $95 \alpha$ and CDR3 $\beta$ Y $97 \beta$ side chains into notches between the MHC $\alpha 1$ and $\alpha 2$ helices and the peptide, 

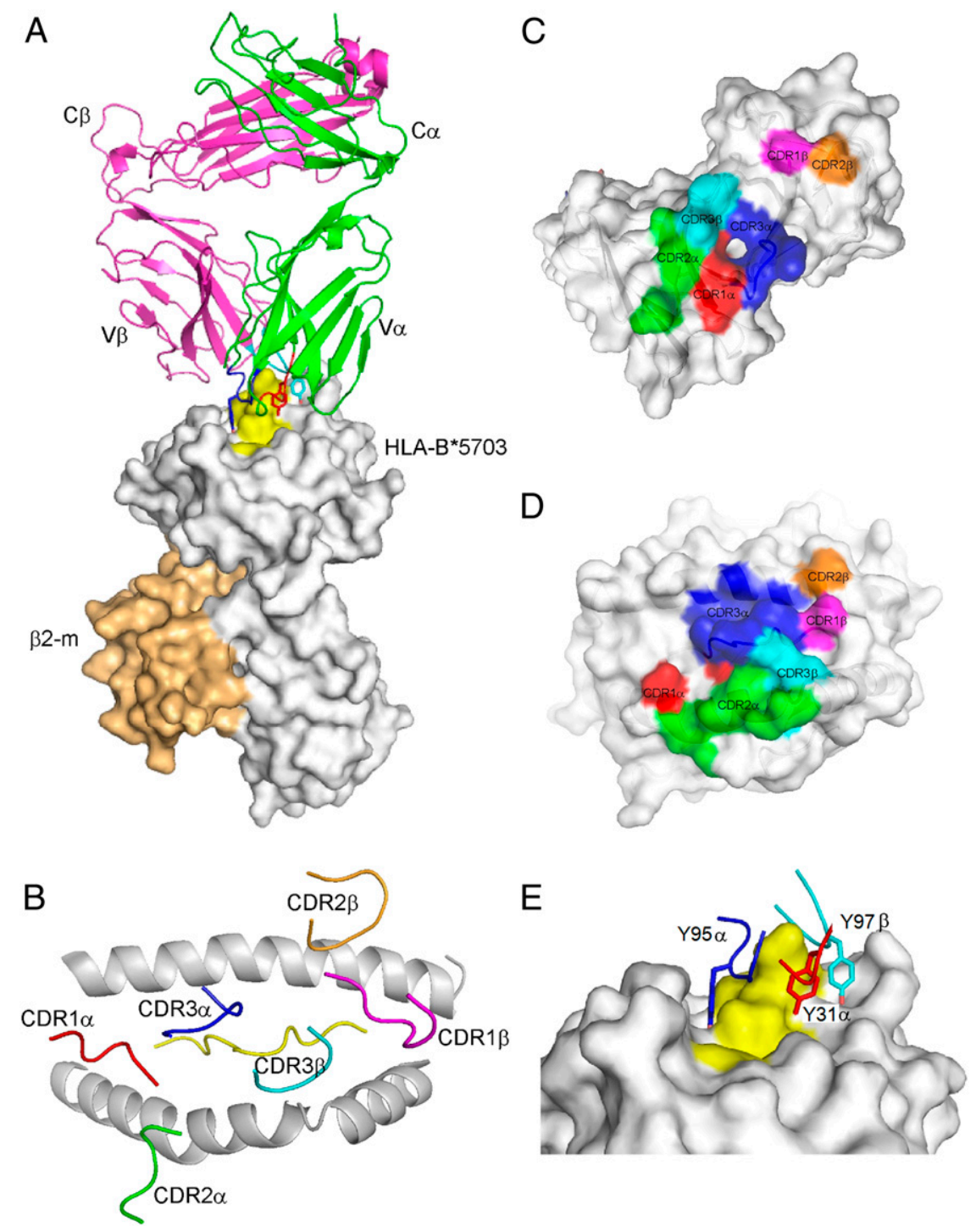

Fig. 1. Structure of the AGA1-HLA-B*5703-KF11 complex. (A) Overview of the AGA1-HLA-B*5703-KF11 complex, the TCR $\alpha$-chain (cyan), the TCR $\beta$-chain (magenta), the HLA-B*5703 heavy chain (gray), $\beta 2$-microglobulin ( $\beta 2-\mathrm{m})$ (orange), and the KF11 peptide (yellow). (B) Positions of CDR loops on HLA-B*5703KF11. The $\alpha$-helices of HLA-B*5703 (gray) and CDR1 $\alpha$ (red), CDR2 $\alpha$ (green), CDR3 $\alpha$ (blue), CDR1 $\beta$ (magenta), CDR2 $\beta$ (orange), and CDR3 (cyan) are shown. (C) Surface representation of the AGA1 TCR residues contacting PMHC, colored as in $B$. $(D)$ Surface representation of pMHC residues contacting AGA1 TCR CDR loops, colored as in $B$. (E) Surface representation of $B \star 5703$ (gray) and KF11 peptide (yellow) and the three CDR loops showing the Tyr side chains clustering around the peptide bulge, colored as described in $B$.

respectively (Fig. 1E). The Y "clamps" are anchored into position by hydrogen bonds between the tyrosine hydroxyl groups and MHC side-chain atoms. The notch occupied by the CDR3 Y97 $\beta$ side chain resembles that described for the highly conserved, public CDR3 R99 $\beta$ side chain in the HLA-A*0201-MP-JM22 complex (38). In the AGA1 complex, Y95 $\alpha$ and Y97 $\beta$ generate van der Waals contacts and good shape complementarity to peptide. The two CDR3 Y side chains, in conjunction with aromatic residues CDR1 $\alpha$ Y31 and Y33 and CDR2 $\alpha$ Y48 and F50, confer a significant proportion of the total BSA (33\%) to the HLA-B*5703-KF11 complex.

Conformational Change of the KF11 Peptide Induced on TCR Recognition. The high resolutions of the HLA-B*5701 and HLA-B*5703 unliganded structures (1.95 $\AA$ and $1.65 \AA$, respectively) allowed many ordered water molecules to be included in the refined structures (Tables 1-5). The structures of the peptides in the two B*57-KF11 subtype complexes are highly superimposable (Fig. $2 A$ and $B$ ), although there are small divergences near the polymorphic MHC residues at positions 114 and 116. In both structures, the peptide buckles to position the central amino acids (4-9) out of the groove, presenting a peptide arch for TCR recognition (Fig. $2 C$ and $D$ ). In contrast to longer peptides such as the EBV 13mer presented in the context of HLA-B*3508, the structures of KF11 presented by both HLA-B*57 subtypes have well-ordered electron density throughout and are clearly defined (9).

The HLA-B*5703-KF11 peptide undergoes conformational change on TCR binding, crumpling into the PBG (Fig. $2 A, B, E$, and $F$ ) and forming a total of nine new hydrogen bonds (Fig. 3). A large cavity between the TCRs CDR $2 \alpha,-3 \alpha$, and $-3 \beta$ accommodates the bulged portion of the peptide. The KF11 conformational changes optimize its contacts with the TCR but also allow the formation of specific contacts between TCR and the MHC helices (Fig. $3 A-D$ ). These conformational readjustments also reposition the crest of the peptide bulge toward the MHC $\alpha 2$ helix (Fig. $2 B$ and $F$ ). The insertion of the side chain of CDR $3 \alpha$ Y 95 between the peptide and $\alpha 1$ helix facilitates the formation of hydrogen bonds to 
Table 2. Direct interactions ( $\leq 4.0 \AA$ A $)$ between the AGA1 TCR and HLA-B*5703

\begin{tabular}{|c|c|c|}
\hline Element & TCR contact & HLA B*5703 contact \\
\hline V $\alpha$ CDR1 & T30 & L163 \\
\hline V $\alpha C D R 1$ & Y31 & Q155 \\
\hline $\mathrm{V} \alpha \mathrm{CDR} 2$ & Y48 & R151, E154 \\
\hline VoCDR2 & F50 & E154, Q155, A158 \\
\hline $\mathrm{V} \alpha \mathrm{CDR} 2$ & N52 & A158, E161, G162 \\
\hline $\mathrm{V}_{\alpha} \mathrm{CDR} 2$ & K67 & E166 \\
\hline$V_{\alpha} C D R 3$ & Y95 & N66, A69, S70, T73 \\
\hline V $\beta C D R 2$ & 151 & E76 \\
\hline V $\beta C D R 3$ & S96 & A150, R151 V152, E154, Q1 \\
\hline VBCDR3 & Y97 & R151 V152, E154, Q155 \\
\hline
\end{tabular}

Table showing the MHC and AGA1 TCR amino acids involved in direct interactions relating to van der Waal's interactions and hydrogen bonding. The cutoff distance for van der Waal's interactions is $\leq 4 \AA$. TCR numbering according to Arden et al. (59).

the MHC side chains N66 and S70 and is accompanied by reorientation of KF11 with the formation of three new hydrogen bonds between the side chain of MHC Q155 and the main-chain atoms of the peptide bulge (Fig. $3 A, B$, and $E$ ). The CDR3 $\beta$ Y97 side chain slots between the peptide and $\alpha 2$ helix, forming hydrogen bonds to the $\alpha 2$ helix and van der Waals contacts to the peptide.

Notably, on TCR binding, the peptide P9 side chain reorients $\sim 80^{\circ}$ into the groove, causing the polymorphic residue Y116 to reposition $\sim 15^{\circ}$ about the $c \alpha / \mathrm{c} \beta$ bond (Figs. $2 F$ and 4 and Fig. S1). Substantial peptide conformational changes were also seen by Tynan et al. (10), who demonstrated movement of a bulged peptide toward the $\alpha 2$ helix to facilitate binding of the ELS4 TCR; however, in that pMHC-TCR complex the peptide bulge was not fully enveloped by the TCR CDR loops.

The CDR3 loop Y side chains that form the peptide clamp are stabilized by three inter-CDR3 hydrogen bonds between the side chain Y99 $\beta$ and the Q96 $\alpha$ carbonyl and between Q96 $\alpha$ and the main-chain atoms of $\mathrm{G} 101 \alpha$ and $\mathrm{G} 103 \alpha$ (Fig. 3D). Comparison of the crystallographic B-factors across the length of the peptide in the unliganded and TCR-bound forms reveals greater disorder at the crest of the peptide in the unliganded state, particularly in the peptide side chain of E6. Therefore, in addition to conformational change, the mobility of the bulged part of the peptide is reduced on TCR binding because of the formation of numerous specific recognition contacts.

Structural Basis for Public Use of Vo5.1. The CDR1 $\alpha$ residues Y31 and Y33 make hydrogen bonds directly to peptide residues $\mathrm{p} 4$ and

Table 3. Direct interactions ( $\leq 4.0 \AA$ A ) between the AGA1 TCR and the KF11 peptide

\begin{tabular}{|c|c|c|}
\hline Element & TCR contact & Peptide contact \\
\hline $\mathrm{V}_{\alpha} \mathrm{CDR} 1$ & Y31 & F3, S4, P5, E6 \\
\hline $\mathrm{V}_{\alpha} \mathrm{CDR} 1$ & Y33 & $\mathrm{E} 6$ \\
\hline $\mathrm{V}_{\alpha} \mathrm{CDR} 3$ & S92 & E6 \\
\hline$V_{\alpha} \mathrm{CDR} 3$ & G93 & S4 \\
\hline$V_{\alpha} C D R 3$ & G94 & S4, P5, E6 \\
\hline $\mathrm{V}_{\alpha} \mathrm{CDR} 3$ & Y95 & S4, P5, E6, 18 \\
\hline $\mathrm{V}_{\alpha} \mathrm{CDR} 3$ & Q96 & E6 \\
\hline $\mathrm{V} \beta \mathrm{CDR} 1$ & D30 & M10 \\
\hline $\mathrm{V} \beta \mathrm{CDR} 3$ & S96 & V7 \\
\hline $\mathrm{V} \beta \mathrm{CDR} 3$ & Y97 & E6, V7 \\
\hline
\end{tabular}

Table showing the peptide atoms involved in direct interactions with the AGA1 TCR including van der Waal's interactions and hydrogen bonding The cutoff distance for van der Waal's interactions is $\leq 4 \AA$. TCR numbering according to Arden et al. (59). p6 (Fig. $3 F$ ), and CDR2 $\alpha$ residues Y48 and F50 contact the HLAB*5703 $\alpha 2$ helix side chains R151 and E154, which undergo a conformational change on TCR binding (Fig. $3 C$ ). The combinations of CDR1 $\alpha$ Y31/Y33 and CDR2 $\alpha$ Y48/F50 are unique to $\mathrm{V} \alpha 5.1$. Therefore, both peptide-specific and MHC-specific contacts generated via different CDR framework loops are created by residue combinations exclusive to $\mathrm{V} \alpha 5.1$, and thus this germline TCR $V \alpha$ framework is specifically optimized to recognize the HLA-B*57 molecule and the unusual arched structure of the bound KF11 peptide. The V $\beta 19$ framework is coselected with the $\mathrm{V} \alpha 5$ but makes minimal contact with the pMHC. The basis for public coselection of V $\beta 19$ could relate to conferring an optimized $\mathrm{V} \beta$ scaffold to support the recognition of KF11 by the V $\alpha 5$ CDR contacts, as previously observed for the JM22-HLA-A*0201-MP complex in which the coselected $\mathrm{V} \alpha 10.2$ domain stabilizes the public CDR $3 \beta$ via water-mediated hydrogen bonds with CDR1 $\alpha$ residues unique to $\mathrm{V} \alpha 10.2$ (38).

Role of Buried Water and Polymorphic Residues in TCR Recognition. The HLA-B*5703 peptide-binding groove floor has four $\beta$-sheetderived tyrosine residues (Y7, Y9, Y99, and Y116), the "Y bed," that hydrogen bond the peptide directly (Y7 and Y99) or are involved in hydrogen bonding the water network between the peptide and the $\mathrm{MHC}$ (Y9 and polymorphic Y116). Of the nine water molecules found in the unliganded HLA-B*5701-KF11 structure, seven are conserved in the unliganded HLA-B*5703-KF11 complex and form a common water network (Fig. $4 A$ and $B$; see Table $\mathrm{S} 1$ for detailed comparisons of the polymorphic $114 / 116 \mathrm{~F}$ pocket contacts). The presence of the bulky Y116 side chain excludes two water molecules in the HLA-B*5703 PBG which are readily accommodated by HLA-B*5701 because of the smaller side chain of $S$ at position 116 . In the unliganded HLA-B*5701-KF11 complex, these water molecules form a water-mediated hydrogen-bonding network between S116-D114 and S116-Y74. On AGA1 binding, KF11 crumples into the HLA-B*5703 groove and forms a new set of contacts with the bound water structure and the underlying $\mathrm{MHC}$ residues. The peptide $\mathrm{P} 9$ side chain reorients into the groove (Fig. $4 C$ ), repositioning the polymorphic side chain of Y116 by $15^{\circ}$ in a domino effect. Although the resolution of the TCR-pMHC complex $(2.4 \AA)$ is not sufficient to position all water molecules buried under the peptide, the reorientation of P9 upon TCR binding is predicted to displace a water molecule, common to the unliganded HLA-B*5701 and B*5703 subtypes (Z48 and A2193, respectively). For HLA-B*5703, the reorientation of Y116 also dislodges a second water molecule (A2195) that hydrogen bonds to the $\mathrm{OH}$ atom of Y116 in the unliganded $\mathrm{B} * 5703-\mathrm{KF} 11$ complex (Fig. $4 C$ ). Structural comparisons indicate that the presence of $\mathrm{S}$ at position 116 in HLA-B*5701 would provide additional space for peptide P9 to reorient into the groove without conformational rearrangement of the MHC p116 sidechain (Fig. 4D). Thus, although both HLA-B*5701-KF11 and HLA-B*5703-KF11 can accommodate an identical binding mode for AGA1, the impact on the bound water structure and the side-chain conformation of Y116 in the HLA-B*5703 PBG must differ from that on the bound water structure linking S116 and D114 in HLA-B*5701, suggesting that there are differences in the kinetics of AGA1 binding for HLAB*5701-KF11 versus B*5703-KF11.

Dissecting the Contributions of the Polymorphic Amino Acids for AGA1 Binding. To test the structure-based prediction of polymorphismdependent differences in TCR recognition described above, we performed kinetic and thermodynamic surface plasmon resonance studies to evaluate the interaction between the AGA1 TCR and the KF11 peptide bound to HLA-B*5701, HLA-B*5703, and the single-hybrid mutants HLA-B*5703 ${ }_{\text {Y116S }}$ and HLA-B*5701 ${ }_{\text {S116Y }}$ (Table 6 and Fig. S2). The primary difference was that AGA1 bound B*5703-KF11 with an approximately fivefold lower affinity as a result of an approximately fivefold faster rate of dissociation 
Table 4. Hydrogen bond interactions between the AGA1 TCR and HLA-B $* 5703$

\begin{tabular}{lccc} 
Element & TCR contact & HLA-B*5703 contact & Length (Å) \\
\hline V $\alpha$ CDR2 & Y48 OH & R151 NH2 & 2.9 \\
V $\alpha$ CDR2 & Y48 OH & E154 OE2 & 2.8 \\
V $\alpha$ CDR3 & Y95 OH & N66 OD1 & 2.7 \\
V $\alpha$ CDR3 & Y95 OH & S70 OG & 3.0 \\
V $\beta C D R 3$ & S96 OG & A150 O & 2.8 \\
V $\beta$ CDR3 & Y97 OH & R151 O & 2.8 \\
V $\beta$ CDR3 & Y97 OH & Q155 N & 2.9 \\
\hline
\end{tabular}

Table showing the MHC and AGA1 TCR atoms involved in hydrogen bonding. TCR numbering according to Arden et al. (59).

$\left(k_{\text {off }}\right)$ than measured for the HLA-B*5701-KF11-AGA1 TCR interaction (Table 6). Interestingly, AGA1 TCR binding to the hybrid mutants was similar to that of HLA-B*5703, and the presence of either or both residues had the same affect on binding affinity and kinetics. This result suggested that these residues are energetically coupled. Further support for this finding was obtained by thermodynamic analysis, which revealed that HLA-B*5703 and the single-mutant HLA-B*5703 ${ }_{\text {Y116S }}$ and HLA-B*5701 $1116 \mathrm{Y}$ variants had similar signatures of TCR dissociation that differed from HLA-B*5701 [HLA-B*5701 $k_{\text {off: }} \Delta \mathrm{H}=25.205 / \Delta \mathrm{G}=19.194$ / $\mathrm{T} \Delta \mathrm{S}=6.011 \mathrm{kcal} \mathrm{mol}^{-1}$ versus HLAB$* 5703 k_{\text {off: }} \Delta \mathrm{H}=20.216 / \Delta \mathrm{G}=$ $18.278 / \mathrm{T} \Delta \mathrm{S}=1.938 \mathrm{kcal} \mathrm{mol}^{-1}$ (see Fig. S2 for details relating to the single-hybrid mutants)]. This finding is consistent with structural data indicating that both residues participate in a network of bonds in the F pocket that accommodates solvent as well as a region of the peptide that is displaced upon TCR binding. It is likely that weaker binding of the AGA1 TCR to HLA-B5703KF11 arises directly or indirectly from these changes. One possibility is that this network of bonds stabilizes the peptide in a more favorable bound conformation in the AGA1/HLA-B*5701KF11 interaction.

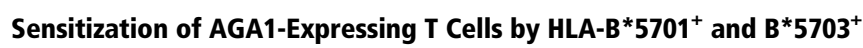
Antigen-Presenting Cells. We also performed functional studies to compare the ability of HLA-B $* 5701^{+}$and $\mathrm{B}^{*} 5703^{+}$target cells to sensitize AGA1-expressing $\mathrm{CD}{ }^{+}$T-cell clones in vitro. IFN- $\gamma$ production and the expression of the lysosomal-associated membrane protein (LAMP) marker CD107a/b on AGA1-expressing Tcell clones was assessed using allogeneic HLA B5701 ${ }^{+}$and B*5703 ${ }^{+}$ B-cell lines (BCLs) expressing exogenously (peptide-pulsed) or endogenously derived [recombinant vaccinia virus (rVV)-infected] KF11 peptide. Experiments were performed for shorter periods to circumvent cumulative effects arising from protracted incubation. In all instances, irrespective of the route of antigen acquisition, antigen-presenting cells expressing HLA-B*5701 demonstrate superior sensitization of AGA1-expressing T-cell clones in vitro (Fig. $5 A-C$ ).

Table 5. Hydrogen bond interactions between the AGA1 TCR and the KF11 peptide

\begin{tabular}{lccc} 
Element & TCR contact & Peptide contact & Length (Å) \\
\hline$V_{\alpha}$ CDR1 & Y31 OH & S4 OG & 2.4 \\
$V_{\alpha}$ CDR1 & Y33 OH & E6 OE2 & 2.6 \\
$V_{\alpha}$ CDR3 & S92 OG & E6 OE1 & 2.6 \\
$V_{\alpha}$ CDR3 & S92 OG & E6 OE2 & 2.6 \\
$V_{\alpha}$ CDR3 & G93 O & S4 OG & 2.6 \\
$V_{\alpha}$ CDR3 & Y95 N & P5 O & 2.8 \\
$V_{\alpha}$ CDR3 & Q96 N & E6 OE1 & 2.8 \\
\hline
\end{tabular}

Table showing the AGA1 TCR and peptide atoms involved in hydrogen bonding. TCR numbering according to Arden et al. (59).

\section{Discussion}

MHCI subtype-specific micropolymorphisms that permit the presentation of shared epitopes are known to impact T-cell selection differentially, reflecting their disparate associations with viral control and susceptibility to autoimmune diseases $(3,4)$. When minor polymorphic differences result in minimal peptide/MHCI conformational differences, the mechanisms underpinning differential $\mathrm{T}$-cell use remain unclear. To investigate these mechanisms, we solved the structure of an 11mer HIV-1 epitope presented by HLA-B*5701, compared this with our previously reported structure of the epitope when presented by the closely related HLA-B*5703 subtype (36), and determined the structure of the TCR in complex with HLA-B*5703-KF11, which in the absence of a HLA-B*5701-KF11-AGA1 cocrystal complex was used as a model to outline the fine molecular details underpinning differential TCR recognition when KF11 is presented by these subtypes in vivo.

We describe a pMHC-TCR cocrystal complex comprising an HIV-1-derived epitope restricted by HLA-B*57 subtypes consistently associated with slower progression to AIDS. AGA1 is a highaffinity TCR $(<1 \mu \mathrm{M})$, at the upper end of the spectrum described for human TCR-pMHCI interactions (39). This TCR frequently is expanded in HLA-B*5701 ${ }^{+}$individuals and demonstrates good cross-reactivity against common escape variants, including those associated with higher viral load, but is absent in carriers of HLAB*5703 who carry a higher burden of KF11 variants in vivo.

Binding of the AGA1 TCR to HLA-B*5703-KF11 is characterized by a dominant $V \alpha$ chain contribution and a high peptide:MHC BSA. The V $\beta$ chain CDRs contribute less substantially to peptide binding but participate in MHC $\alpha 2$ recognition. The AGA1 TCR uses a distinct clamp, comprising Y residues contributed by CDR $1 \alpha$, $\mathrm{CDR} 3 \alpha$, and CDR3 $\beta$ motifs, that envelopes the crest of the peptide bulge, wedging their side chains into notches in the MHC $\alpha 1$ / $\alpha 2$-peptide interface and forming many of the pMHC contacts conferring specificity to the interaction. The CDR3s are characteristically short; the Y clamp allows access around the arched peptide, facilitating recognition of the molecular surface without requiring long CDR main chains and also provides the TCR with a flexible CDR surface allowing the conformational adjustments required to accommodate the arched peptide on TCR binding. This mode of recognition, involving the insertion of CDR side chains into notches between the MHC and peptide cavities ("pegs filling notches"), was described initially for JM22 TCR recognition of the "featureless" influenza HLA-A*0201 restricted MP epitope (38) and a second example recently was reported for TCR binding to a bulged epitope (10). The addition of a third example, as described here, suggests that this mode of binding has broad utility in terms of TCR recognition.

The HLA-B*5703-KF11-AGA1 complex has features in common with the SB27 and ELS4 TCR complexes, the few examples for which structural data relating to the recognition of atypically long peptides have been described $(9,10)$. Binding of the SB27 TCR to the centrally arched HLA-B*3508-restricted LPEP peptide represents an unusual interaction in which the $\mathrm{V} \alpha$ chain dominates the pMHC interface, the peptide arch remains prominent on TCR ligation, and the exceptionally few TCR-mediated MHC contacts generated. In common with the SB27 TCR, binding of the AGA1 TCR is characterized by a substantial contribution of the peptide and TCR $\alpha$-chain to the BSA and a focus on the Nterminal half of the peptide. However, in contrast to HLA-B*3508LPEP, in which the peptide bulge becomes less disordered upon SB27 ligation, the KF11 epitope is relatively ordered in the unliganded state and undergoes substantial conformational change on TCR binding, although this is less extensive than the bulldozing of the malleable B*3501-EPLP epitope by the ELS4 TCR. Also, unlike the SB27 TCR, AGA1 encompasses a substantial MHC recognition footprint resembling that generated by the ELS4 
A

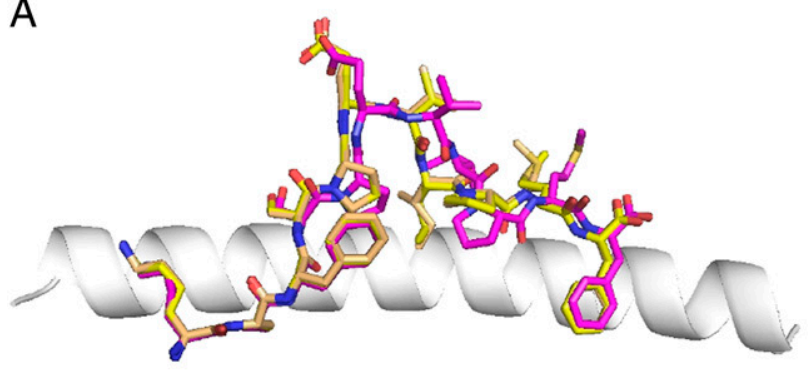

B
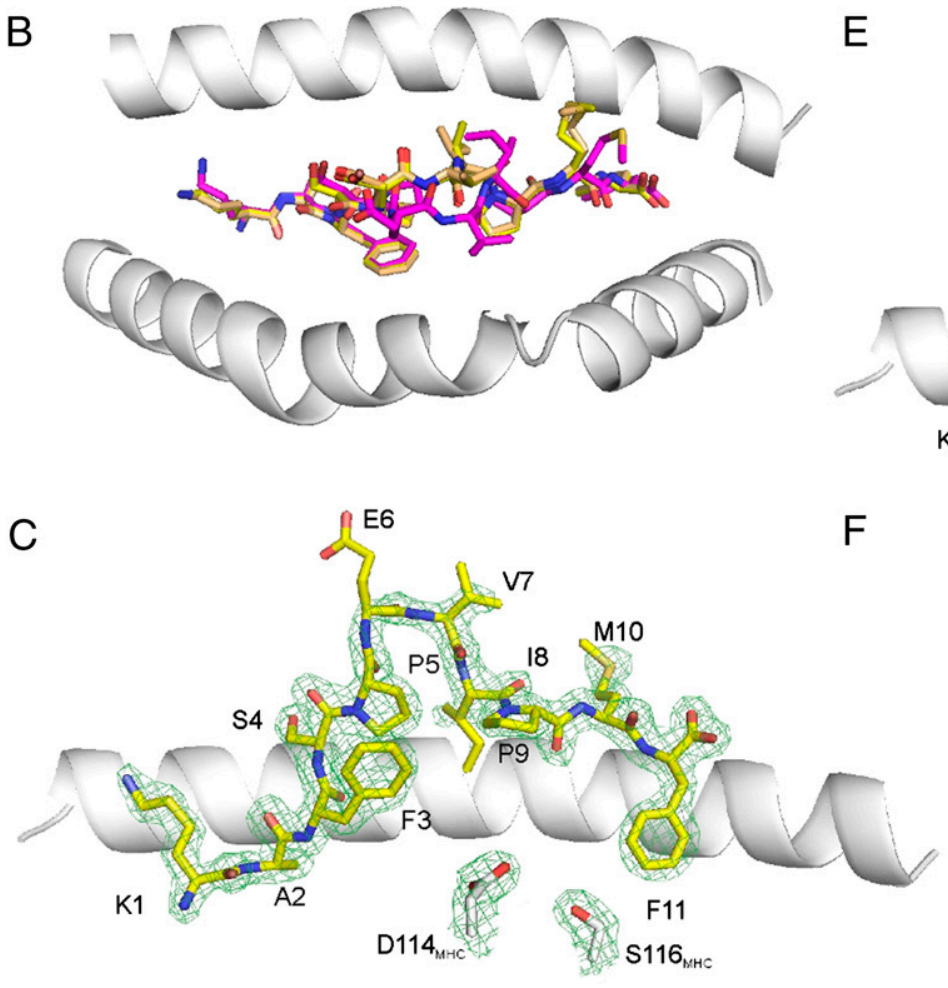

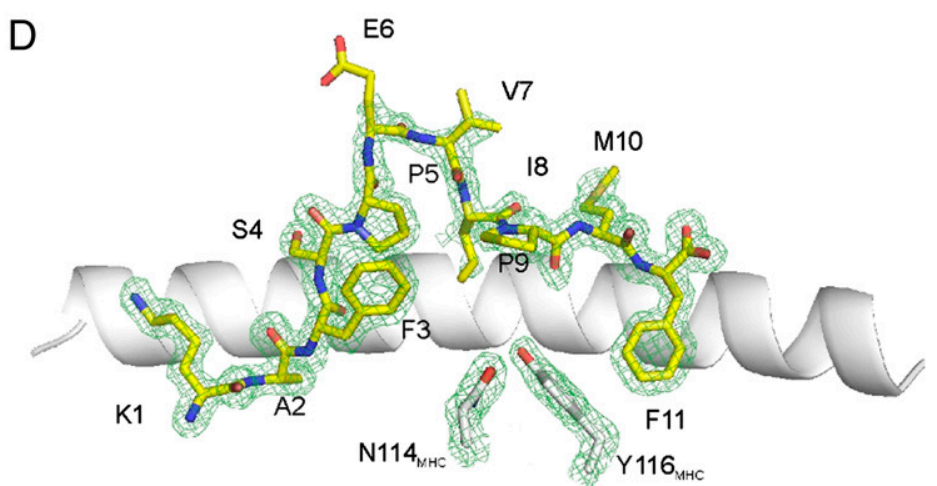

E

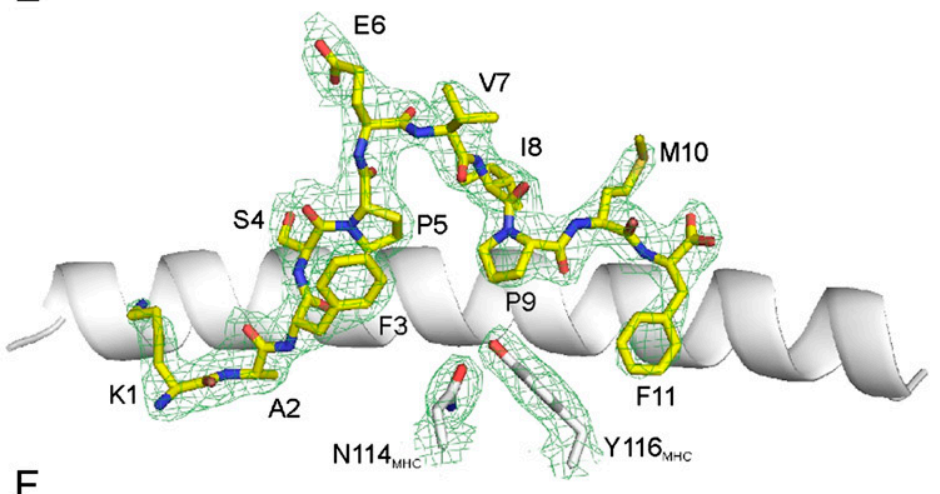

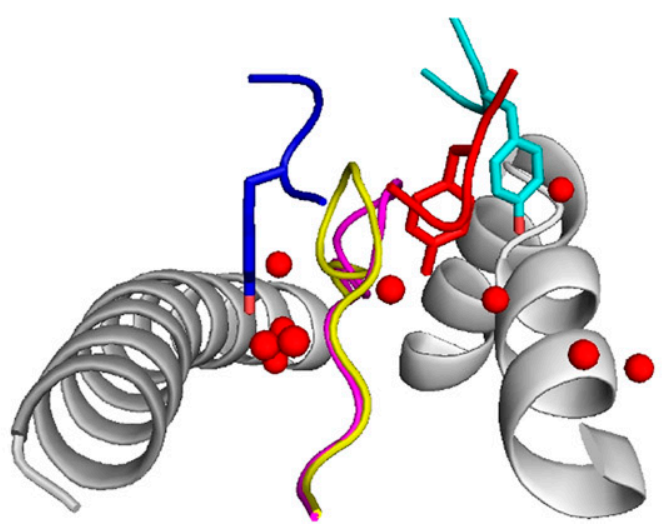

Fig. 2. KF11 peptide structures in HLA-B*5703 and HLA-B*5701. (A) Profile view of superimposition via the HLA peptide-binding grooves for KF11 peptides in HLA-B*57, showing conformational change between the unliganded peptide structures, with HLA-B*5701-KF11 (orange), HLA-B*5703-KF11 (yellow), and AGA1 TCR-bound peptide (magenta) illustrated. The MHC $\alpha 1$ helix is shown in gray. (B) Top view of $A$, showing both MHC $\alpha 1$ and $\alpha 2$ helices (gray). (C-E) Refined peptide structures and polymorphic side chains at MHC position 114 and 116 and associated 2fo-fc electron density maps are shown for the HLA$B * 5701-K F 11(C)$, HLA-B*5703-KF11 $(D)$, and HLA-B*5703-KF11-AGA1 TCR $(E)$ complexes. $(F)$ MHC superimposition showing the position of unliganded HLA$B * 5703$ KF11 peptide (yellow) versus AGA1 TCR-bound peptide (magenta) water molecules displaced on TCR binding HLA-B*5703-KF11 (red spheres) and the positions of the three CDR loops and associated Tyr side chains, colored as described in Fig. $1 A$.

TCR. However, in contrast to both the SB27 and ELS4 TCRs, the pMHCI-binding interface formed by AGA1 is dominated by the $\alpha$-chain contribution in relation to the number of specific peptide contacts generated. Many of these contacts originate from germline-encoded CDR $1 \alpha$ and $\mathrm{J} \alpha$ residue side chains, highlighting an underlying structural basis for $\mathrm{V} \alpha 5$ chain usage. This principle is shared with the JM22 TCR, in which binding to HLA-A*0201$\mathrm{MP}$ is characterized by the dominant role of the germline-encoded side chains of CDR1 $\beta$ and CDR2 $\beta$ loop residues in contacting the peptide (38). Despite these inherent similarities, the recognition interfaces presented by HLA-A*0201-MP (flat) and HLAB*5703-KF11 (bulged) represent dramatically opposed structural landscapes, and our findings further reiterate the diverse binding modes used by TCRs when peptides are presented in conformationally distinct formats.

The frequency of $\alpha$ - and $\beta$-chain production constitutes a major determinant driving biased TCR chain use, particularly in relation to $\beta$-chain use, in vivo $(40,41)$. Production frequency is influenced both by the number of nucleotide additions incorporated at the $\mathrm{V}$ D-J boundary during TCR chain rearrangement, with the frequency of production reduced with increasing numbers of nucleotide additions, and by convergent amino acid use, where production frequency correlates positively with the incorporation of nucleotides that converge to encode identical amino acid motifs (41). The AGA1 TCR is highly conserved and selected in unrelated HLAB*5701 ${ }^{+}$individuals and because the hypervariable CDR3 loops are short, should represent frequently generated TCR chains and 

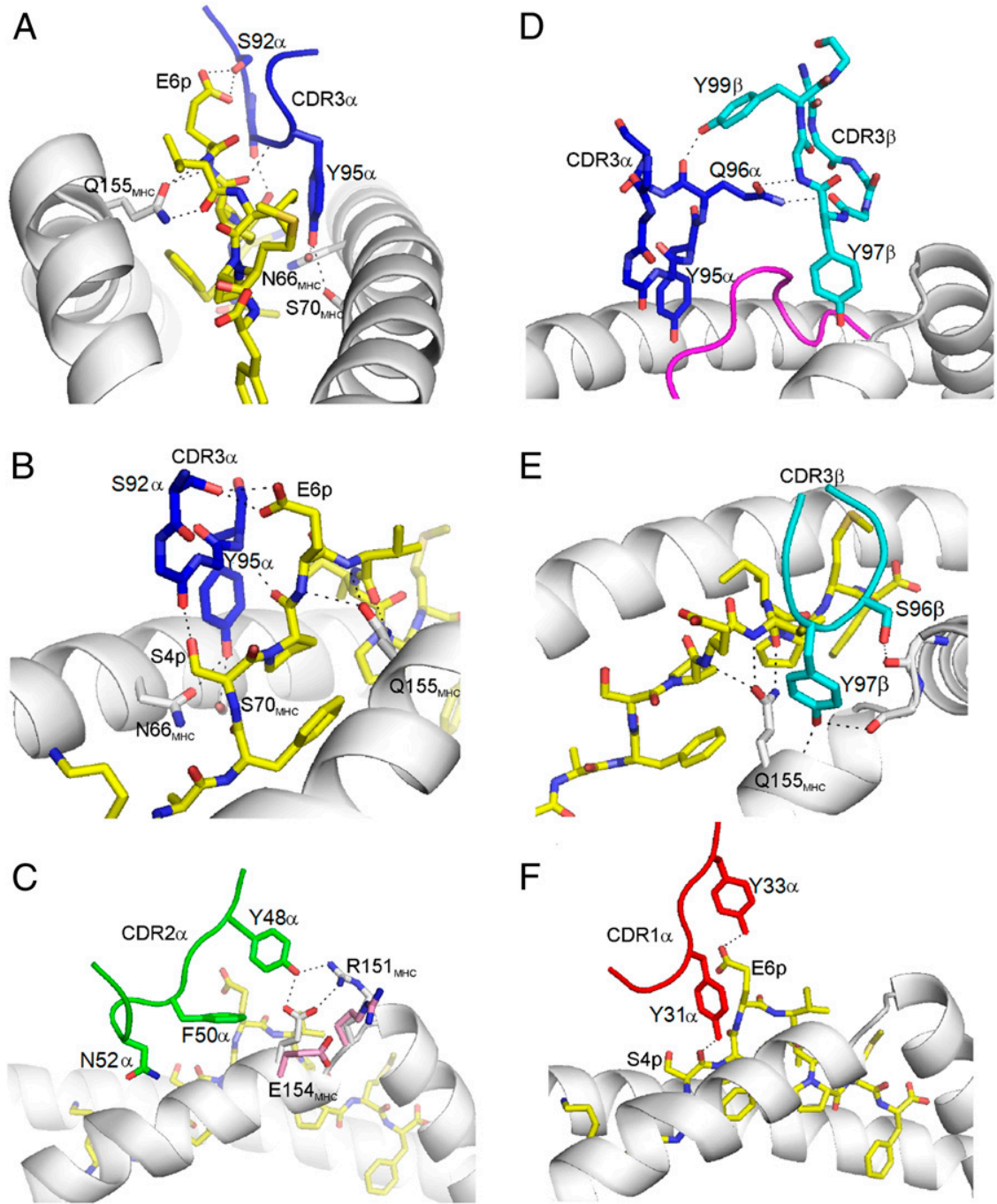

Fig. 3. TCR CDR loop interactions with HLA-B*5703-KF11. (A) The CDR3 loop (blue), HLA-B*5703 (gray), KF11 peptide (yellow), and hydrogen bonds (dashed lines) are displayed. (B) Alternative view of $A$. (C) The CDR2 $\alpha$ loop (green), HLA-B*5703 (gray), KF11 peptide (yellow), and hydrogen bonds (dashed lines) are shown. (D) The CDR3 $\alpha$ and CDR3 $\beta$ loops (blue and cyan, respectively), HLA-B $* 5703$ (gray), KF11 peptide (magenta), and hydrogen bonds between loops (dashed lines) are shown. (E) The CDR3 $\beta$ loop (cyan), HLA-B*5703 (gray), KF11 peptide (yellow), and hydrogen bonds (dashed lines) are shown. (F) The CDR1 $\alpha$ loop (red), HLA-B $\$ 5703$ (gray), KF11 peptide (yellow), and hydrogen bonds (dashed lines) are illustrated. Residues are identified as one-letter amino acids followed by the AGA1 TCR chain ( $\alpha$ or $\beta$ ) or peptide ( $p$ ).

a common $\alpha \beta$ TCR pair in vivo. The AGA1 CDR3 $\alpha$ and $-\beta$ chain loops include few N-terminal additions, particularly the CDR3 $\alpha$ loop which comprises germline-encoded residues almost exclusively (21). The non-germline segments demonstrate interindividual nucleotide diversity but converge to encode identical amino acid motifs. However, despite a predicted high precursor frequency, there is a noted lack of AGA1 TCR use in response to KF11 in HLA-B $* 5703^{+}$individuals. We propose that HLA-B*57 subtypespecific structural features could constitute a key determinant limiting use of AGA1 by the HLA-B*5703 subtype in vivo. These polymorphic specific effects involve subtle structural alterations, whereby the peptide changes induced on TCR binding culminate in adjustments of MHC side chains and the bound water molecules within the PBG. Water molecules play an integral role in $\mathrm{MHCI}$ peptide binding, and although strictly conserved water molecule networks contribute to hydrogen bonding the peptide $\mathrm{N}$ and $\mathrm{C}$ termini in the $\mathrm{A}$ and $\mathrm{F}$ binding pockets, bound water molecules in the central region of the $\mathrm{PBG}$, in conjunction with the polymorphic residues, can contribute to the plasticity of peptide binding for individual MHCI molecules (42). Differences in both the density and distribution of peptide-MHC groove water networks have been implicated in facilitating the reorientation and flexibility of centrally positioned residues of an epitope presented by the closely related HLA-B*2705 and B*2709 subtypes (4). For the structural analyses presented here the differences in the positioning of central KF11 residues in the HLA-B*5701 and B*5703 unliganded complexes are minimal (rmsd $<0.3 \AA$ ), but water molecule networks coupled to polymorphic MHC residues in the PBG are impacted differently by conformational changes in the KF11 peptide required for TCR binding and hence indirectly affect the properties of TCR ligation. Therefore, for certain TCR-pMHCI subtype pairs, minor variations of buried water molecules networks in association with polymorphic residues may subtly shape $\mathrm{T}$-cell repertoire use in vivo.

Binding studies revealed that AGA1 bound all pMHCI variants with comparable association rate constants $\left(k_{\text {on }}\right)$, indicating that the polymorphic differences did not radically alter the initial binding phase. This finding is also supported by our structural observations, which illustrate that the primary TCR contacts are focused on peptide residues $(\mathrm{p} 4-\mathrm{p} 7)$ in a region not structurally modified by the polymorphisms in the unliganded state. The 
A
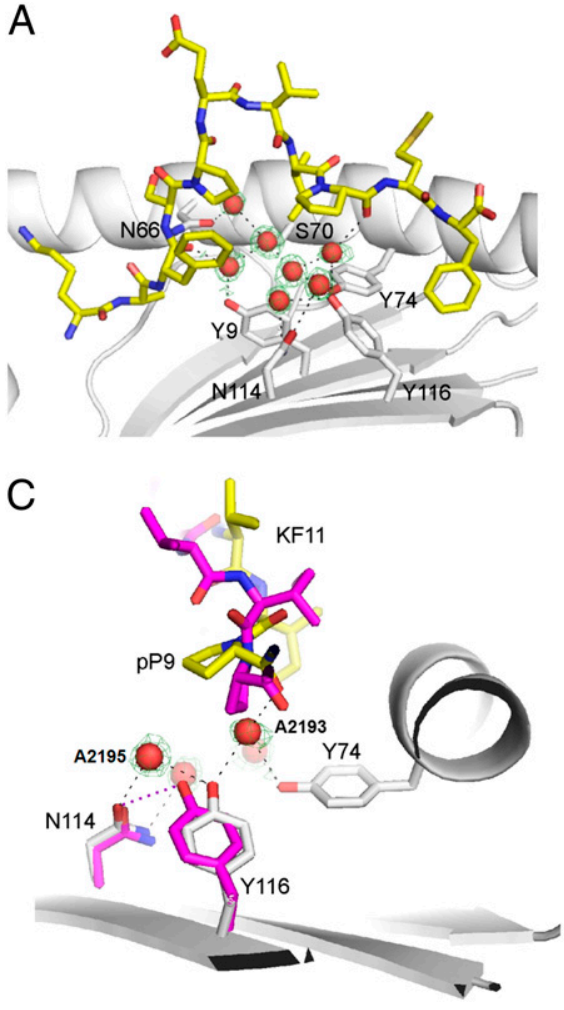

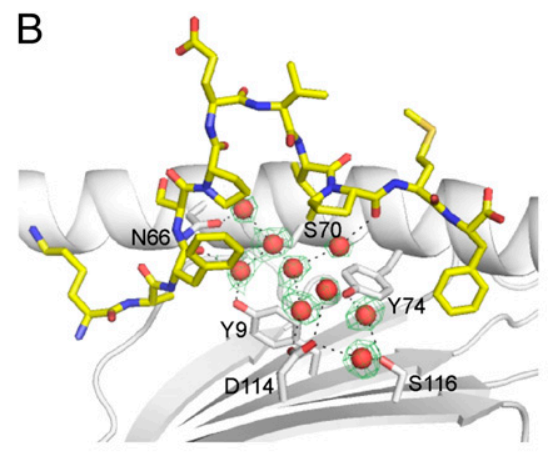

D

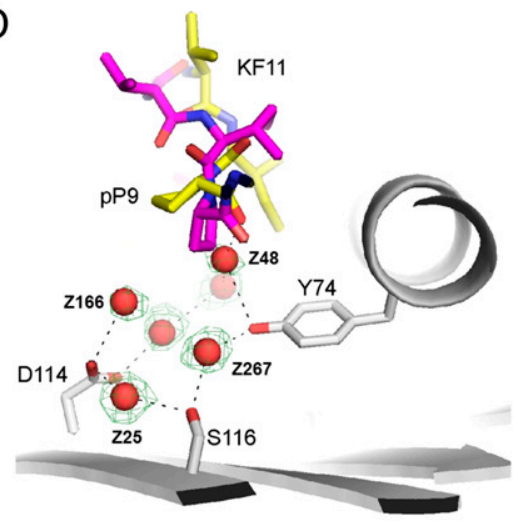

Fig. 4. Ordered water molecules and conformational changes on TCR binding. $(A)$ The re-refined unliganded HLA-B*5703-KF11 structure: water molecules (red spheres) contoured with 2 fo-fc electron density at $1 \sigma, H L A-B * 5703$ (gray), KF11 peptide (yellow), and hydrogen bonds (dashed lines). (B) Unliganded HLA-B*5701-KF11 structure: water molecules (red spheres) contoured with $2 \mathrm{fo}-\mathrm{fc}$ electron density at $1 \sigma$, HLA-B*5701 (gray), KF11 peptide (yellow), and hydrogen bonds (dashed lines). (C) HLA-B*5703-KF11-AGA1 TCR complex structure (magenta) superimposed with HLA-B*5703-KF11, colored as in $A$. Peptide P9 side chain from the TCR complex sterically clashes with water molecule A2193 and polymorphic MHC Y116, reorienting Y116 by some $15^{\circ}$ and thereby making a new hydrogen bond to the $\mathrm{N} 114$ side chain (magenta dashed line). (D) HLA-B*5703-KF11-TCR complex structure (magenta) superimposed with HLA$B * 5701-K F 11$, colored as in $B$. The peptide P9 side chain from the TCR complex is predicted to sterically clash with water molecule Z48 that hydrogen bonds to Y74. greatest discrepancies were reflected in the off-rates $\left(k_{\text {off }}\right)$, which corroborated our structure-based observations that the impact of the subtype-specific polymorphisms arise as a consequence of and subsequent to AGA1 ligation and shape the kinetics of receptor occupancy. Interestingly, the p114 and p116 HLA-B*57 polymorphisms had the same effect when introduced individually as when both were present (in HLA-B*5703), and the nonadditive effect of these mutations suggests that both residues are involved in the same energetically favorable network. Our structural data indicate that these residues participate in the same network of solvent-protein interactions in the PBG pocket that accommodates conformational adjustments in the peptide induced upon binding. One possibility is that this network of bonds stabilizes the peptide in a more favorable bound conformation in the AGA1/ HLA-B*5701-KF11 complex and that either mutation alone can disrupt this interaction network.

The IMGT/HLA Database (http:/www.ebi.ac.uk/ipd/imgt/hla/) lists 57 putative HLA-B*57 subtypes, the majority of which fall into categories expressing either a $\mathrm{D}_{114}(\mathrm{Q}) \mathrm{S}_{116}$ or $\mathrm{N}_{114}(\mathrm{Q}) \mathrm{Y}_{116}$ motif. The conserved coupling of these dimorphisms suggests a functional significance, presumably allowing subtle differences in their capacity to sample antigen repertoires (3), interact with components of the peptide loading complex such as tapasin (43), and present distinct epitopes (44). It has been suggested that the polymorphic differences separating these HLA-B*57 subtypes subtly modify their peptide-binding repertoires (45), and these modifications could have implications intrathymically, producing shifts in T-cell repertoire selection and the availability of the peripheral AGA1-expressing T cells in HLA-B*5703 ${ }^{+}$individuals. Likewise, the presence of MHC class I allotypes that interfere with thymic selection of AGA1-expressing T cells could promote their depletion in carriers of HLA-B*5703, a phenomenon originally described to explain the lack of the naturally dominant HLA-B*08-restricted LC13 TCR in individuals who coexpress HLA-B*08 and HLA-B*4402 (46). Finally, genetic factors specific to the TCR loci that dictate the frequency of AGA1 $\alpha$ - and $\beta$-chain production cannot be ruled out as a contributing determinant (47). However, in absence of specific evidence relating to the lack of peripheral AGA1-expressing cells in HLA$\mathrm{B}^{*} 5703^{+}$individuals, it is possible that the polymorphic differences separating the HLA-B*57 subtypes do not notably alter their circulating frequencies but promote differential use of AGA1expressing $\mathrm{T}$ cells via the subtle molecular mechanism described here. Although the affinity of the AGA1 TCR for HLA-B*5703KF11 matches other agonist interaction, $T$ cells expressing this receptor might be out-competed by higher affinity clonotypes on a HLA-B* 5703 background. This choice could contribute, in part, to the different frequencies of circulating KF11 variants associated with elevated viral load in these patient groups. Our findings might also provide an explanation for the association of HLA-B*2705 with ankylosing spondylitis, as the single $\mathrm{F}$ pocket polymorphic difference distinguishing it from the nonsusceptible $B^{*} 2709$ subtype

Table 6. Affinity and kinetics of AGA1 binding

\begin{tabular}{lcccc} 
Immobilized ligand (in complex with KF11) & Analyte & $k_{\text {on }}\left(\times 10^{4} \mathrm{M} / \mathrm{s}\right)$ & $\mathrm{k}_{\text {off }}\left(\mathrm{s}^{-1}\right)$ & $\mathrm{K}_{\mathrm{D}}(\mu \mathrm{M})$ \\
\hline HLA-B $* 5701$ & AGA1 & 8.3 & 0.05 & 0.63 \\
HLA-B*5703 & AGA1 & 8.5 & 0.25 & 3.0 \\
HLA-B*5701 ${ }_{\mathrm{S} 116 \mathrm{Y}}$ & AGA1 & 5.4 & 0.28 & 5.2 \\
HLA-B*5703Y116s & AGA1 & 8.1 & 0.22 & 2.7 \\
\hline
\end{tabular}

Binding constants were measured by surface plasmon resonance at $25^{\circ} \mathrm{C}$. 
A

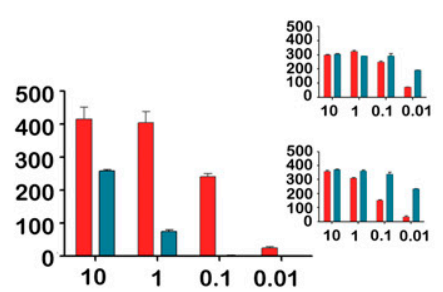

吊
B

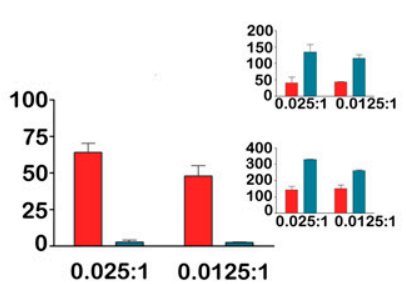

C
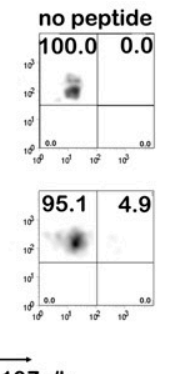

$\longrightarrow$ CD107a/b

Fig. 5. Enhanced sensitization of AGA1-expressing T-cell clones by $B * 5701$-expressing antigen-presenting cells. $\left(A\right.$ and $B$ ) AGA1-expressing CD $8^{+} T$-cell clones and clones demonstrating KF11 specificity but expressing diverse $\alpha \beta T C R$ pairs (Insets) were tested for their ability to secrete IFN- $\gamma$ in response to peptidepulsed HLA-B*5701- (red) and HLA-B*5703-expressing (teal) BCL targets, over a 10-fold peptide titration from 10-0.01 $\mu \mathrm{M}(A)$ and IIIB gag rVV-infected targets at effector:target (E:T) ratios of 0.025:1 and 0.0125:1 (B). CD8 ${ }^{+}$T-cell responses are reported as the number of spot forming cells (SFC) per 500 cell input; values shown are median \pm SEM. (C) Expression of the CD107a/b LAMP markers in response to peptide-pulsed HLA-B*5701 and B*5703 (1 and $10 \mu M$, respectively) at an E:T ratio of 2:1, by flow cytometry analysis. $\mathrm{CD}^{+} \mathrm{T}$ cells were gated as a function of both positive (forward scatter versus side scatter) and negative (forward scatter versus CD8) gating. CD8 ${ }^{+}$cells are indicated along the $y$-axis and CD107a/b expression along the $x$-axis. A representative of three independent experiments (each performed in triplicate) is presented.

could differentially impact TCR repertoire selection rather than driving epitope-binding specificity or conformational changes (4).

Collectively, our structural, thermodynamic, and functional data suggest that use of clonotypes expressing the AGA1 TCR constitutes an optimized choice when KF11 is presented on a HLAB*5701 background. This preference reflects a mechanism involving TCR-induced peptide conformational readjustments, modulation of PBG water networks, and indirect interactions with polymorphic MHCI residues. Very subtle differences, such as the polymorphic changes described here, could presumably alter the balance of the responding $\mathrm{T}$ cell repertoire, with underestimated but important implications for TCR usage in vivo.

\section{Methods}

Protein Production and Purification. The soluble form of the $\mathrm{V} \beta 19 \mathrm{~V} \alpha 5$ (AGA1) TCR was refolded from inclusion bodies as described previously (48). Mutations were introduced into this sequence by overlapping PCR using complementary primers engineered to contain the desired mutation, and the TCR $\alpha$ and $-\beta$ products were cloned into the PET $22 \mathrm{~b}(+)$ vector (Novagen). The $\alpha$-chain was cloned in frame with the C-terminal hexahistidine tag from the vector. TCR chains were expressed using the Escherichia coli strain BLR (Novagen), isolated as inclusion bodies, purified, resolubilized, and refolded as described. The refolded complexes were purified by anion exchange, $\mathrm{Ni}^{+2}$, and gel-filtration chromatography. Receptor quality was confirmed by chromatographic movement and nonreduced and reduced SDS/PAGE profiles.

The peptide KAFSPEVIPMF (KF11) was refolded with the HLA-B*5701, HLA$B * 5703$, and single-mutant hybrid HLA-B*57 subtype heavy chains (HLA$B * 5703_{Y 116 S}$ and $H L A-B * 5701_{S 116 Y}$ ) with a $C$-terminal biotinylation tag and $\beta_{2} M$ as described (38). For crystallography HLA-B*57 subtypes without a biotinylation tag were refolded.

Surface Plasmon Resonance. Surface plasmon resonance studies were performed using a BIAcore 3000 (GE Healthcare) as previously described $(49,50)$. C-terminal biotinylated pMHC was immobilized via covalently coupled streptavidin at levels of 1,100-1,400 resonance units. Binding kinetics of the AGA1 TCR was not substantially limited by mass transfer at these immobilization levels (50). The TCR was injected over multiple flow cells, and the binding response was determined by subtracting the response measured on a control flow cell containing no pMHC from the response measured in flow cells containing $\mathrm{PMHC}$. Affinity and kinetic parameters were determined as previously described $(49,50)$. Thermodynamic parameters were determined by measuring the dependence of the affinity and kinetics on temperature (51).

Crystallization and Data Collection. All crystallizations were performed using the sitting-drop vapor-diffusion technique, typically using drops containing 2 $\mu \mathrm{L}$ of protein solution and $2 \mu \mathrm{L}$ of reservoir solution. Single AGA1-HLA B*5703KF11 complex crystals grew as rods with dimensions up to $500 \times 120 \times 20 \mu \mathrm{m}$ at room temperature from a stoichiometric ratio of pMHC:TCR at a final concentration of $10 \mathrm{mg} / \mathrm{mL}$ in $11 \%$ (vol/vol) PEG8000, $50 \mathrm{mM} \mathrm{Mes,} \mathrm{pH} \mathrm{7.2.} \mathrm{Single}$ crystals of the HLA B*5701-KF11 complex grew as plates with dimensions up to $200 \times 150 \times 30 \mu \mathrm{m}$ from $16 \%$ PEG8000, $50 \mathrm{mM}$ Mes, $\mathrm{pH} 6.5$, at room temperature. Crystals were soaked briefly and sequentially in reservoir solutions containing $10 \%$ and $20 \%$ glycerol and then were flash-cooled and maintained at $100 \mathrm{~K}$ in a cryostream (Oxford Cryosystem). Datasets were collected on station ID14.2 at the European Synchrotron Radiation Facility (Grenoble, France) equipped with an ADSC-q4 CCD detector (Area Detector Systems Corporation). Details of the crystal space groups and unit cells are given below and in Table 1. Datasets were auto-indexed and integrated with the program DENZO followed by scaling with the program SCALEPACK (52).

Structure Determination and Refinement. All structures were determined by molecular replacement using the CCP4 program (53) Phaser (54). For the AGA1-HLA-B*5703-KF11 complex the molecular-replacement solution was obtained using the HLA-B*5703-KF11 structure (2BVO) and a model of the AGA1 TCR based on 1OGA TCR (55). The unliganded HLA-B*5701-KF11 structure was determined by molecular replacement using the 2BVO HLA$B * 5703-K F 11$ with peptide omitted as an initial search model, and subsequently the KF11 peptide was built into Fo-Fc difference density maps.

For all structures, rounds of refinement were done using a restrained TLS refinement algorithm in REFMAC5 (56). Manual refitting of the models was carried out using Coot (57), and in the final stages of refinement, water picking was performed with ARPw/ARP (58). Almost all regions of the models' densities were clear. Table S1 was produced using Pymol (DeLano Scientific LLC) and the statistics package Origin, version 5.0 (OriginLab Corporation).

IFN- $\gamma$ ELISpot Assay. The standard ELISpot assay was used to detect IFN- $\gamma$ release by $C D 8^{+}$T-cell clones, but with modifications (30). In brief, 10,000 peptidepulsed HLA-B* $5701^{+}$and $B * 5703^{+}$immortalized $B C L s$ were incubated with 500 $\mathrm{CD}^{+}$T-cells from clones expressing the AGA1 TCR or from HLA-B*5701-restricted clones demonstrating KF11 specificity but expressing distinct $\alpha \beta T C R$ pairs. When recognition of endogenously derived KF11 was evaluated, nonautologous $\mathrm{HLA}-\mathrm{B} 5701^{+}$and $\mathrm{B} * 5703^{+} \mathrm{BCL}$ targets were infected with $\mathrm{rVV}$ expressing the HIV-1 IIIBgag gene (VP1287) at a multiplicity of infection of 5, in serum-free medium for $90 \mathrm{~min}$ at $37^{\circ} \mathrm{C}$. The vP1287 vaccinia virus was obtained through the AIDS Research and Reference Reagent Program, National Institute of Allergy and Infectious Diseases, National Institutes of Health. Following extensive washing, rVV-infected BCLs were recovered in serum-containing medium for $60 \mathrm{~min}$ at $37^{\circ} \mathrm{C}$. ELISpot assays were prepared using 20,000 and 40,000 rVV-infected cells as targets and 500 effector T-cell clones and were set up in triplicate. Assays were harvested following $8 \mathrm{~h}$ incubation. Results are reported as the number of spot-forming cells (SFCs) per 500 T-cell input, and SFCs double the number observed with medium alone were considered positive.

CD107a/b Expression. CD107a/b staining was performed as previously described but with slight modifications (21). Fifty thousand T-cell clones were incubated with allogeneic HLA-B*5701+ and $B * 5703^{+} B C L$ s a ratio of 2:1, either pulsed with KF11 peptide (to a final concentration of 1 and $10 \mu \mathrm{M}$ ) or incubated with medium alone, and in the presence of $5 \mu \mathrm{L}$ of CD107a and $-b$ FITC-labeled monoclonal antibodies at $37{ }^{\circ} \mathrm{C}$ (BD Biosciences). After $1 \mathrm{~h}$ incubation, GolgiStop (BD Biosciences) was added to individual samples at 
a concentration recommended by the manufacturer. After a total of $5 \mathrm{~h}$ at $37{ }^{\circ} \mathrm{C}$, cells were stained with anti-hCD8-PerCP (BD Biosciences) and fixed in $2 \%$ formaldehyde-PBS before flow cytometry analysis using a FACSCalibur (BD Biosciences) and FlowJo v7 software (TreeStar)

1. Kaslow RA, et al. (1996) Influence of combinations of human major histocompatibility complex genes on the course of HIV-1 infection. Nat Med 2(4):405-411.

2. Carrington M, et al. (1999) HLA and HIV-1: Heterozygote advantage and $B * 35-\mathrm{CW}^{*} 04$ disadvantage. Science 283(5408):1748-1752.

3. Messaoudi I, Guevara Patiño JA, Dyall R, LeMaoult J, Nikolich-Zugich J (2002) Direct link between mhc polymorphism, $T$ cell avidity, and diversity in immune defense. Science 298(5599):1797-1800.

4. Hülsmeyer M, et al. (2002) HLA-B27 subtypes differentially associated with disease exhibit subtle structural alterations. J Biol Chem 277(49):47844-47853.

5. Goulder PJ, Watkins DI (2008) Impact of MHC class I diversity on immune control of immunodeficiency virus replication. Nat Rev Immunol 8(8):619-630.

6. Hülsmeyer M, et al. (2004) Dual, HLA-B27 subtype-dependent conformation of a selfpeptide. J Exp Med 199(2):271-281.

7. Archbold JK, et al. (2009) Natural micropolymorphism in human leukocyte antigen provides a basis for genetic control of antigen recognition. J Exp Med 206(1):209-219.

8. Tynan FE, et al. (2005) The immunogenicity of a viral cytotoxic T cell epitope is controlled by its MHC-bound conformation. J Exp Med 202(9):1249-1260.

9. Tynan $\mathrm{FE}$, et al. (2005) T cell receptor recognition of a 'super-bulged' major histocompatibility complex class I-bound peptide. Nat Immunol 6(11):1114-1122.

10. Tynan FE, et al. (2007) A T cell receptor flattens a bulged antigenic peptide presented by a major histocompatibility complex class I molecule. Nat Immunol 8(3):268-276.

11. Kloverpris HN, et al. (2012) HLA-B*57 Micropolymorphism shapes HLA allele-specific epitope immunogenicity, selection pressure, and HIV immune control. $J$ Virol 86(2): 919-929.

12. Bell MJ, et al. (2009) The peptide length specificity of some HLA class I alleles is very broad and includes peptides of up to 25 amino acids in length. Mol Immunol 46(8-9): 1911-1917.

13. Burrows SR, Rossjohn J, McCluskey J (2006) Have we cut ourselves too short in mapping CTL epitopes? Trends Immunol 27(1):11-16.

14. Klein MR, et al. (1994) Associations between HLA frequencies and pathogenic features of human immunodeficiency virus type 1 infection in seroconverters from the Amsterdam cohort of homosexual men. $J$ Infect Dis 169(6):1244-1249.

15. Migueles SA, et al. (2000) HLA B*5701 is highly associated with restriction of virus replication in a subgroup of HIV-infected long term nonprogressors. Proc Natl Acad Sci USA 97(6):2709-2714.

16. Costello C, et al. (1999) HLA-B $\star 5703$ independently associated with slower HIV-1 disease progression in Rwandan women. AIDS 13(14):1990-1991.

17. Marsh SGE, Parham P, Barber LD (2000) The HLA Factsbook (Academic, London).

18. Altfeld M, et al. (2003) Influence of HLA-B57 on clinical presentation and viral control during acute HIV-1 infection. AIDS 17(18):2581-2591.

19. Altfeld $M$, et al. (2006) HLA Alleles Associated with Delayed Progression to AIDS Contribute Strongly to the Initial CD8(+) T Cell Response against HIV-1. PLoS Med 3(10): e403

20. Crawford H, et al. (2009) Evolution of HLA-B*5703 HIV-1 escape mutations in HLA $B \star 5703$-positive individuals and their transmission recipients. J Exp Med 206(4):909921.

21. Gillespie GM, et al. (2006) Strong TCR conservation and altered T cell cross-reactivity characterize a B*57-restricted immune response in HIV-1 infection. J Immuno/ 177(6): 3893-3902.

22. Leslie AJ, et al. (2004) HIV evolution: CTL escape mutation and reversion after transmission. Nat Med 10(3):282-289.

23. Draenert $\mathrm{R}$, et al. (2004) Immune selection for altered antigen processing leads to cytotoxic T lymphocyte escape in chronic HIV-1 infection. J Exp Med 199(7):905-915

24. Brumme ZL, et al. (2008) Marked epitope- and allele-specific differences in rates of mutation in human immunodeficiency type 1 (HIV-1) Gag, Pol, and Nef cytotoxic Tlymphocyte epitopes in acute/early HIV-1 infection. J Virol 82(18):9216-9227.

25. Crawford $\mathrm{H}$, et al. (2007) Compensatory mutation partially restores fitness and delays reversion of escape mutation within the immunodominant HLA-B $\star 5703$-restricted Gag epitope in chronic human immunodeficiency virus type 1 infection. $J$ Viro/ 81(15): 8346-8351.

26. Kiepiela $P$, et al. (2007) CD8+ T-cell responses to different HIV proteins have discordant associations with viral load. Nat Med 13(1):46-53.

27. Yu XG, et al. (2007) Mutually exclusive T-cell receptor induction and differentia susceptibility to human immunodeficiency virus type 1 mutational escape associated with a two-amino-acid difference between HLA class I subtypes. J Virol 81(4): 1619-1631.

28. Bailey JR, Williams TM, Siliciano RF, Blankson JN (2006) Maintenance of vira suppression in HIV-1-infected HLA-B*57+ elite suppressors despite CTL escape mutations. J Exp Med 203(5):1357-1369.

29. Turnbull EL, et al. (2006) HIV-1 epitope-specific CD8+ T cell responses strongly associated with delayed disease progression cross-recognize epitope variants efficiently. J Immunol 176(10):6130-6146.

30. Gillespie GM, et al. (2002) Cross-reactive cytotoxic T lymphocytes against a HIV-1 p24 epitope in slow progressors with $B * 57$. AIDS 16(7):961-972.
ACKNOWLEDGMENTS. We thank the patients who participated in this study the clinicians and research staff who collected the clinical samples, and the staff of ID14 eh2 at the European Synchrotron Radiation Facility for support during X-ray data collection.

31. Simons BC, et al. (2008) Despite biased TRBV gene usage against a dominant HLA B57restricted epitope, TCR diversity can provide recognition of circulating epitope variants. J Immunol 181(7):5137-5146.

32. Green KJ, et al. (2004) Potent T cell response to a class I-binding 13-mer viral epitope and the influence of HLA micropolymorphism in controlling epitope length. Eur $J$ Immunol 34(9):2510-2519.

33. Probst-Kepper M, et al. (2004) Conformational restraints and flexibility of 14-meric peptides in complex with HLA-B*3501. J Immunol 173(9):5610-5616.

34. Speir JA, Stevens J, Joly E, Butcher GW, Wilson IA (2001) Two different, highly exposed, bulged structures for an unusually long peptide bound to rat MHC class RT1-Aa. Immunity 14(1):81-92.

35. Hörig H, Young AC, Papadopoulos NJ, DiLorenzo TP, Nathenson SG (1999) Binding of longer peptides to the $\mathrm{H}-2 \mathrm{~Kb}$ heterodimer is restricted to peptides extended at their $\mathrm{C}$ terminus: Refinement of the inherent MHC class I peptide binding criteria. J Immuno 163(8):4434-4441

36. Stewart-Jones GB, et al. (2005) Structures of three HIV-1 HLA-B*5703-peptide complexes and identification of related HLAs potentially associated with long-term nonprogression. J Immunol 175(4):2459-2468.

37. Rudolph MG, Stanfield RL, Wilson IA (2006) How TCRs bind MHCs, peptides, and coreceptors. Annu Rev Immunol 24:419-466.

38. Stewart-Jones GB, McMichael AJ, Bell Jl, Stuart DI, Jones EY (2003) A structural basis for immunodominant human T cell receptor recognition. Nat Immuno/ 4(7):657-663.

39. Cole DK, et al. (2007) Human TCR-binding affinity is governed by MHC class restriction. J Immunol 178(9):5727-5734.

40. Venturi $V$, et al. (2006) Sharing of T cell receptors in antigen-specific responses is driven by convergent recombination. Proc Natl Acad Sci USA 103(49):18691-18696.

41. Venturi V, Price DA, Douek DC, Davenport MP (2008) The molecular basis for public T-cell responses? Nat Rev Immunol 8(3):231-238.

42. Smith KJ, et al. (1996) Bound water structure and polymorphic amino acids act together to allow the binding of different peptides to MHC class I HLA-B53. Immunity 4(3):215-228

43. Williams AP, Peh CA, Purcell AW, McCluskey J, Elliott T (2002) Optimization of the MHC class I peptide cargo is dependent on tapasin. Immunity 16(4):509-520.

44. Zernich D, et al. (2004) Natural HLA class I polymorphism controls the pathway of antigen presentation and susceptibility to viral evasion. J Exp Med 200(1):13-24.

45. Chessman D, et al. (2008) Human leukocyte antigen class I-restricted activation of $\mathrm{CD} 8+\mathrm{T}$ cells provides the immunogenetic basis of a systemic drug hypersensitivity. Immunity 28(6):822-832.

46. Burrows SR, Khanna R, Burrows JM, Moss DJ (1994) An alloresponse in humans is dominated by cytotoxic T lymphocytes (CTL) cross-reactive with a single Epstein-Bar virus CTL epitope: Implications for graft-versus-host disease. J Exp Med 179(4): $1155-1161$.

47. Posnett DN, et al. (1994) Level of human TCRBV3S1 (V beta 3) expression correlates with allelic polymorphism in the spacer region of the recombination signal sequence. J Exp Med 179(5):1707-1711.

48. Boulter JM, et al. (2003) Stable, soluble T-cell receptor molecules for crystallization and therapeutics. Protein Eng 16(9):707-711.

49. Lee JK, et al. (2004) T cell cross-reactivity and conformational changes during TCR engagement. J Exp Med 200(11):1455-1466.

50. Willcox BE, et al. (1999) TCR binding to peptide-MHC stabilizes a flexible recognition interface. Immunity 10(3):357-365.

51. Davis-Harrison RL, Armstrong KM, Baker BM (2005) Two different T cell receptors use different thermodynamic strategies to recognize the same peptide/MHC ligand. $J \mathrm{Mo}$ Biol 346(2):533-550.

52. Otwinowski ZM, Minor W (1997) Processing of X-ray diffraction data collected in oscillation mode. Methods Enzymol 276:307-326

53. Anonymous; Collaborative Computational Project, Number 4 (1994) The CCP4 suite: Programs for protein crystallography. Acta Crystallogr D Biol Crystallogr 50(Pt 5): 760-763.

54. McCoy AJ, Grosse-Kunstleve RW, Storoni LC, Read RJ (2005) Likelihood-enhanced fast translation functions. Acta Crystallogr D Biol Crystallogr 61(Pt 4):458-464.

55. Arnold K, Bordoli L, Kopp J, Schwede T (2006) The SWISS-MODEL workspace: A web-based environment for protein structure homology modelling. Bioinformatics 22(2):195-201.

56. Murshudov GN, Vagin AA, Dodson EJ (1997) Refinement of macromolecular structures by the maximum-likelihood method. Acta Crystallogr D Biol Crystallogr 53 (Pt 3):240-255.

57. Emsley P, Cowtan K (2004) Coot: Model-building tools for molecular graphics. Acta Crystallogr D Biol Crystallogr 60(Pt 12 Pt 1):2126-2132.

58. Morris RJ, Perrakis A, Lamzin VS (2002) ARP/wARP's model-building algorithms. I. The main chain. Acta Crystallogr D Biol Crystallogr 58(Pt 6 Pt 2):968-975.

59. Arden B, Clark SP, Kabelitz D, Mak TW (1995) Human T-cell receptor variable gene segment families. Immunogenetics 42(6):455-500. 